\title{
Resource Management in Decentralized Industrial Automated Guided Vehicle Systems
}

\author{
M. De Ryck ${ }^{\mathrm{a}, *}$, M. Versteyhe ${ }^{\mathrm{a}}$, K. Shariatmadar ${ }^{\mathrm{a}}$ \\ ${ }^{a}$ Faculty of Engineering Technology, KU Leuven, \\ Spoorwegstraat 12, 8200 Bruges, Belgium
}

\begin{abstract}
This paper proposes an advanced decentralized method where an Automated Guided Vehicle (AGV) can optimally insert charging stations into an already assigned optimal tour of task locations. In today's industrial AGV systems, advanced algorithms and techniques are used to control the whole fleet of AGVs robustly and efficiently. While in academia, much research is conducted towards every aspect of AGV control. However, resource management or battery management is still one aspect which is usually omitted in research. In current industrial AGV systems, AGVs operate until their resource level drops below a certain threshold. Subsequently, they head to a charging station to charge fully. This programmed behaviour may have a negative impact on the manufacturing systems performance. AGVs lose time charging at inconvenient moments while this time loss could be avoided. Using the approach, an AGV can choose independently when it will visit a charging station and how long it will charge there. A general constrained optimization algorithm will be used to solve the problem and the current industrial resource management will be used as a benchmark. We use a simple extension of the Traveling Salesman Problem (TSP) representation to model our approach. The paper follows a decentral approach which is in the interest of the authors. The result of the proposal is a compact and practical method which can be used in today's operative central or decentral controlled AGV systems.
\end{abstract}

Keywords: Automated Guided Vehicles, Decentralization, Resource Management, Traveling Salesman Problem, Constrained Optimization

\section{Introduction}

Automated Guided Vehicles are mobile robots which perform transportation tasks in all types of applications. From e-commerce warehouses to material handling in assembly lines, to pharmacy, and further. Not only one robot but a whole fleet of mobile robots cooperate to perform an efficient transport of goods.

\footnotetext{
* Corresponding author

Email addresses: matthias.deryck@kuleuven.be (M. De Ryck), mark.versteyhe@kuleuven. be (M. Versteyhe),

keivan.shariatmadar@kuleuven.be (K. Shariatmadar)
}

Since 1954 [1] AGVs are widely deployed in logistical environments all over the globe and are now on the rise in a lot of applications. Current AGV systems incorporate hightechnological features to control the whole fleet of robots most safely and efficiently. That said, resource management is one of the features that remained untouched. However, in future manufacturing, more attention should go to sustainable manufacturing in which resource management is handled more efficiently [2]. In current industrial systems, AGVs perform a specified sequence of transportation tasks between different locations in the operating area. As they move and carry goods, they consume resources. 
If their resource level drops below a certain threshold, the AGVs head to the closest charging station which is located somewhere in the operating area. Subsequently, the AGVs charge until their maximum resource level is reached. This charging behaviour is also used in [3]. While moving towards such a charging station and while charging, the AGVs can of course not execute any tasks. In current manufacturing processes, this may have a negative impact on the overall performance of the system:

- The moment the resource level of an AGV drops below the threshold, the current location of the AGV can, by chance, be far from the closest charging station. Charging a bit earlier at a charging station closer to the initial tour could be a better decision. This would cause a reduction of the total extra travel time needed to head to a charging station and return to execute the remaining tasks.

- The moment the resource level of an AGV drops below the threshold can be at an inappropriate moment. If, for example during a rush order, an AGV needs to charge fully, this takes a lot of time which is maybe unnecessary. An AGV could make a smarter decision to not charge fully but yet being able to complete the tasks of the rush order. After the busy period, the AGV could decide to continue charging.

In general, it should be possible for an AGV to choose whether it includes a charging station in its task sequence by itself, and how long it will stay at this chosen charging station. Using this approach, transportation and resource management in AGV systems could be handled very efficiently. The application potential of this approach could be extended to applications outside the AGV world. Also delivery cars, trucks, or autonomous vehicles in general, need to execute a path of pickup and drop off points. Also they have to refuel from time to time. Our approach can be implemented in these situations in a way that drivers can be advised to better charge at a certain location on their trajectory to save time and provide comfort. Especially in electrical vehicles, this approach can be more beneficial. In general, our approach can be implemented in every resource-based transportation system because of its simplicity and generality.

The paper is organized as follows: Section 2 reviews the current literature on resource management in AGV systems. Section 3 discusses briefly the TSP model where our approach is based on. Section 4 formulates our approach and defines the objectives and constraints. Section 5 defines a simple situation where we will work out some possible solutions manually. In Section 6, our approach will be benchmarked to the currently employed resource management in AGV systems. In Section 7, our approach will be situated in the upcoming decentralized trend in future AGV systems. The whole paper will be concluded in Section 8 .

\section{Literature review}

Although it is a relevant problem where operational efficiency is pushed to its limits, literature on the management of resources is very rare [4]. I. F.A Vis [5] proposed a survey on the design and control of AGV systems where she states that battery management is hardly addressed in AGV research. She recommends researchers to include this resource management into the control of AGV systems in the future. By integrating efficient resource management, the overall system performance could be improved. The results of this management principle could further be used as constraints in scheduling and routing of AGV fleets. Another review on the design and control of AGV systems [6] endorses the same statements that battery management is usually omitted in research. However, R. W. McHaney [7] proved already early on that the performance of AGV systems is clearly influenced by including battery management. He presents three types of charging schemes: 
- Opportunity charging, in which the AGV uses its idle time to charge.

- Automatic recharging, in which an AGV runs until its battery is depleted to a certain level and then goes for charging.

- Combination system, this is a combination of the last two.

M. Ebben [8] also saw the need for efficiently managing resources. In his paper, he suggested four heuristics for routing the AGVs towards a battery station if its resource level drops below a certain threshold:

- Select the closest charging station

- Select the first station on the current route

- Select the farthest reachable battery station on the current route

- Select the battery station that will cause minimum delay considering both travel time and waiting time in a queue

Q. S. Kabir et al. 9] made a comparison between the different routing heuristics for battery management from M. Ebben. They conclude that the heuristic of choosing the battery station which minimizes the total travel time and waiting time when the battery level drops between a certain threshold performs the best. In their paper, they also mention that the most common schemes for battery charging of AGVs are battery swapping and automatic charging. In battery swapping, the AGV swaps its empty battery for a fully charged one. In automatic charging, the AGV charges itself at a station while the battery stays inside the AGV. In another article, Q. S. Kabir et al. [3] explore the possibilities of increasing manufacturing capacities through battery management of AGVs. They investigate how the duration of battery charging can be varied to increase the flexibility of a manufacturing system concluding that a more frequent charging of the battery increases the productivity of a manufacturing system significantly when using lead-acid batteries. Our approach reflects the aforementioned features as minimizing the total travel time is the optimization objective of our approach and also as a more frequent charging results from the approach. From this, we can conclude that implementing our resource management behaviour will benefit the overall performance of the system.

Besides the influence of battery management on the overall system performance, battery management also affects the battery lifecycle. Some charging schemes can cause a faster deterioration of the battery and some schemes can benefit the lifetime of the battery. T. Kawakami et al. [10] wrote an article about the battery life cycle management in which they analyse the effect of the AGV operation modes (load, unload, move, etc.) on the battery life and its deterioration. They propose a methodology for planning a battery strategy that minimizes battery-related costs. According to H. Kiehne [11, a battery should never reach a level lower than $20 \%$, and never a level higher than $80 \%$ to keep up a good battery life-cycle.

Obviously, the influence of battery management on manufacturing performance and on battery lifetime is dependent on the type of batteries used. In [3] Q. S. Kabir et al. state that the most widely used batteries for industrial AGVs are the lead-acid batteries. Agreed by [10], this battery type receives the most of its charge during the initial phase of charging as opposed to the later phase. The best way to keep lead-acid batteries healthy is to avoid over-discharge and insufficient charging. Frequent charging of these batteries extends the battery life. The capacity of lead-acid batteries is significantly less at a higher discharging rate. Other battery types used in AGV systems are nickel-cadmium batteries and lithium-ion batteries. These types have certain advantages over lead-acid batteries (lighter, faster charging time) but are signifi- 
cantly more expensive than lead-acid batteries. For this reason, lead-acid batteries are still the most used battery types in AGV systems.

Besides battery management, there are several other battery-related features which can influence the performance of the system or the lifespan of a battery:

- The number of charging stations available

- The location of these charging stations in the operating area

- The capacity of these stations (amount of AGVs which can charge at the station at the same time)

The aforementioned features are more related to the design of AGV systems rather than the control of it. As the focus of our research is on AGV control specifically, we will not include these design-related features in our research. In the next section, we will review the Traveling Salesman model on which our approach is based on.

\section{Brief formulation of the TSP model}

To model our approach, we will build further on the well-known Traveling Salesman Problem (TSP) [12] representation. The TSP is a mathematical graph theory problem that is used in computer science to model realworld optimization problems and to test optimization algorithms. The problem defines itself as follows: A salesman has the task to visit $n$ cities $x_{1}, \ldots, x_{n}$ starting from a starting city $x_{1}$. The salesman knows all the distances between each city. He has to visit every city exactly once. The salesman has a distance matrix $D=\left\{d_{i j}\right\}$, with $d_{i j}$ the distance from city $i$ to city $j$. He has to visit all the cities exactly once in such a way that the total traveled time is minimized. After he visited all the cities, he has to return to the starting city $x_{1}$. The objective function can be formulated as follows:

$$
\min f(\bar{x})=\sum_{i=1}^{n-1}\left(t_{x_{i}, x_{i+1}}\right)+t_{x_{n}, x_{1}}, \quad \bar{x}=\left(x_{1}, \ldots, x_{n}\right)
$$

In this formula, $\bar{x}$ represents a whole tour which contains every city $x_{i}$ exactly once. $t_{x_{i}, x_{i+1}}$ is the traveled time between city $x_{i}$ and city $x_{i+1}$. A constant speed $v_{c}$ is assumed. The travel time $t_{x_{i}, x_{i+1}}$ is calculated as follows:

$$
t_{x_{i}, x_{i+1}}=\frac{d_{x_{i}, x_{i+1}}}{v_{c}}
$$

$t_{x_{n}, x_{1}}$ is the traveled time between the last city $x_{n}$ and the starting city $x_{1}$. So $f$ is the total time traveled to visit every city exactly once when following the tour $\bar{x}$. The goal of the problem is to find a tour $\bar{x}$ which minimizes this objective function $f$.

The optimized tour $\bar{x}$ containing cities the salesman has to visit will be the input to our approach. We assume that the AGV starts with an already optimized tour which it received from a central or decentral task allocation algorithm. Our approach builds further on this optimal tour by inserting extra cities if the robot is not able to finish the tour without charging. Based on this optimized tour and the initial resources of the AGV before starting the tour, our approach outputs an optimal insertion of charging stations and the time it will charge at these stations.

\section{Formulation of our approach}

We start from the assumption that the AGV has received a tour $\bar{x}$ from a task allocation algorithm of $n$ stations it has to visit. We also assume that the AGV starts at a depot station $x_{1}$ and that it returns to this station after visiting all the task locations. At the depot station, we assume that the AGV has enough idle time to charge fully. So at the beginning of the tour, the AGV is charged fully $\left(R_{\text {init }}=100 \%\right)$. We also assume that after completing the tour and arriving at the depot station, the AGV 
can again charge fully. A tour $\bar{x}$ can be defined as:

$$
\bar{x}=\left[x_{1}, \ldots, x_{n}\right]
$$

We extend the Traveling Salesman Problem where the salesman has to visit each city with $m$ resource cities which the AGV does not have to visit necessarily. It has the opportunity to visit these cities (or charging locations) only if it needs to charge. It can also decide how long it will stay in these stations and thus how many resources it will receive. The AGV can thus choose by itself whether it includes a resource station in its initial tour or not. In the following text, we use the term stations instead of cities in the context of AGV system transport. The summation of the number of stations to visit and the number of available resource stations makes a total of $n+m$ stations $c_{i}$ that AGV can visit. The vector $c$ of length $n+m$ which includes all stations can be formulated as follows.

$$
c=\left[c_{1}, \ldots, c_{n+m}\right]
$$

The AGV knows the positions of every station and knows the distances between them. It keeps a distance matrix $D=\left\{d_{i j}\right\}$, with $d_{i j}$ the distance from location $i$ to location $j$. To know which of the stations in $c$ that are actually resource stations, a vector $r c$ is formulated as a binary vector of length $n+m$ denoting which of the stations that are resource stations. The value $r c_{i}$ is 1 if station $c_{i}$ is a resource station.

$$
r c_{i} \in\{0,1\} \quad \forall i \in\{1, \ldots, n+m\}
$$

Matrix $r b c$ with size $(n \times m)$ denotes which resource station will be included and where in the tour. Matrix $r b c$ is a binary matrix where the rows (index $i$ ) represent the stations in the initial tour and the columns (index $j$ ) represent the charging stations. Element $r b c_{i j}=1$ if charging station $j$ is included after station $i$ in the initial tour $\bar{x}$

$$
r b c_{i j} \in\{0,1\} \quad \forall i \in\{1, \ldots, n\}, \forall j \in\{1, \ldots, m\}
$$

A matrix $r t$ with size $(1 \times n)$ is defined to denote how long the robot will stay at each resource station included into a specific position in the initial tour. It is a matrix where element $r t_{i}$ is the time $T$ the AGV will stay at a resource station optionally included after station $i$ in the initial tour $\bar{x}$. $T$ can vary between a minimum charging time $T_{\min }$ and a maximum charging time $T_{\max }$. If the AGV stays for $T_{\max }$, it is fully charged.

$$
r t_{i} \in\left[T_{\min }, T_{\max }\right] \quad \forall i \in\{1, \ldots, n\}
$$

The output of our optimization is a new tour $\overline{\bar{x}}$ which consists of the initial stations to visit and the inserted resource stations. Let $p \leq n+m$ be the length of this new tour (amount of stations to visit $n+$ amount of charging stations included).

As we present an approach on battery management, it is important to define the used battery charging and consuming characteristics. In our approach, we will assume that the AGV has lead-acid batteries as literature shows that these are the most prevalent in AGV systems:

- Charging characteristic: Figure 1 shows a typical charging characteristic of lead-acid batteries.

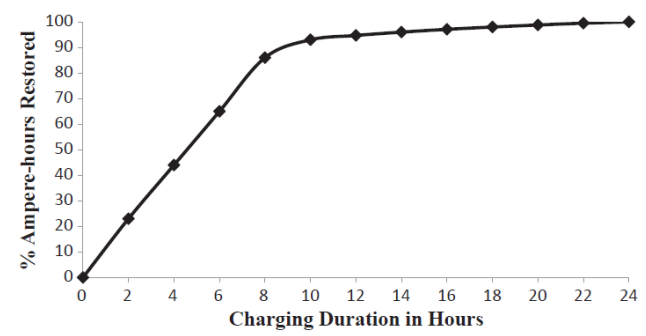

Figure 1: Charging characteristic of lead-acid batteries 3

The graph shows the restored ampere-hours (in \%) in function of the charging duration in hours. The property of this type of batteries is that they gain 
a lot of charge in the beginning period of charging and a little amount in the end. In this first part, where the battery gains most of its charge, the characteristic has a linear trend. For this reason we will approximate the characteristic by the following linear function:

$$
\operatorname{deltaR}_{c h}(T)=\left(\frac{R_{\max }}{T_{\max }}\right) \cdot T
$$

In this function, delta $R_{c h}$ is the amount of resources the AGV has gained. $R_{\max }$ is the maximum amount of resources the AGV can gain $(100 \%)$ and $T_{\max }$ is the time the robot needs to charge fully.

- Consumption characteristic: While the AGV is operating, it is consuming resources. A part of the consumption goes to all the sensors and computing devices. The larger part of the consumption goes to the actuators for driving and material handling. During driving, factors like speed, load the AGV carries, used wheel types, and other factors can influence the resource consumption. This consumption will be different for each of the AGV's actions: being idle, accelerating, braking, braking with inverter motors, driving at a constant speed, loading, unloading, will all cause different resource consumption characteristics. 3] includes an overview of ampere draws for different AGV activities. In reality, the total consumption viewed overtime for the whole tour thus consists of different consumption characteristics dependent on the action performed in a certain time interval. However, for simplicity, our approach will assume that the resource consumption characteristic of the AGV during the whole tour will consist of a single function in time as if the AGV would drive at a constant speed during the whole tour without accelerating, braking, loading, or unloading. The resource consumption delta $R_{c}$, defined as the remaining resource level in time, can thus be formulated as follows:

$$
\operatorname{deltaR}(t)=f(t)
$$

Although implementing on a real vehicle will require a more sophisticated model, for simplicity, we will consider a constant ampere draw for all activities over the whole tour and thus a linear consumption (Ah) in time. This will globally result in a less steep resource consumption function compared to real-live situations. But this does not affect the benefits of our approach: saving time not charging fully and saving time choosing the closest charging station to the initial tour. When comparing our approach to other approaches, this is a good approximation as the other major energy-asking actions, besides driving at a constant speed, will only cause a bias in total resource consumption as the amount and type of pickings and drop-offs will be the same no matter which approach is used. Thus, because we use a fixed initial tour of predefined tasks to execute, this simplification still brings robust results.

The goal of the approach is to minimize the total travel time of the tour including charging times. We can formulate the objective function to be minimized as follows.

$$
f=\sum_{i=1}^{p-1}\left(t_{x_{i}, x_{i+1}}\right)+t_{x_{p}, x_{1}}+\sum_{i=1}^{m}(r t \times r b c)
$$

In this formula $p$ is the amount of stations in the new tour $\overline{\bar{x}}$ with $p \leq n+m,\left(t_{x_{i}, x_{i+1}}\right)$ is the travel time needed to go from the $i$ th station to the $(i+1)$ th station in the tour, $t_{x_{p}, x_{1}}$ is the time to return to the start station, $\sum_{i=1}^{m}(r t \times r b c)$ is the total time the AGV is charging. This is summed over all the stations to visit and all the resource stations.

This objective function is subjected to some linear and nonlinear constraints. Firstly, there cannot be two or more resource stations included between two normal stations on 
the tour at the same time. Therefore, our objective function is subjected to the following linear constraint.

$$
\sum_{j=1}^{m}\left(r b c_{i j}\right) \leq 1 \quad \forall i \in\{1, \ldots, n\}
$$

Secondly, we have a nonlinear resource constraint. A solution will be discarded if there are not enough resources to reach the next station. So our objective function is subjected to the following nonlinear constraint.

$$
\text { resource_at_station }(i) \geq 0 \quad \forall i \in\{1 \ldots, p\}
$$

Vector resource_at_station(), is a vector which contains the available resources at each station on the tour including the charging stations. resource_at_station(1) is the number of available resources if the AGV is at the starting station. This equals the initial amount of resources the AGV has, in the beginning, $\left(R_{\text {init }}\right)$.

Thirdly, an extra nonlinear constraint is added. A solution will be discarded if the resource level exceeds the maximum resource level at any time in the tour. So our objective function is also subjected to the following nonlinear constraint.

$$
\text { resource_at_station }(i) \leq R_{\max } \quad \forall i \in\{1 \ldots, p\}
$$

In the next section, an example will be worked out using a simple situation.

\section{Illustrative example problem}

\subsection{Example problem description}

As a practical example, we take a simple situation with four normal stations $(n=4)$ the AGV has to visit (station $1,2,3$, and 4 ) and one resource station $(m=1)$ the AGV can optionally insert in its tour (station 5), see Figure 2. The locations of all the stations are gathered in vector $c$ with length $(n+m)=5 . c_{1}=(0,0)$ is the depot station.

$$
c=\left[\begin{array}{ccccc}
0 & 0 & 40 & 40 & 20 \\
0 & 40 & 40 & 0 & 20
\end{array}\right]
$$

The distances between all stations are concatenated in distance matrix $D$. The distance between station 1 and station 2 is $D_{12}=40 \mathrm{~m}$.

$$
D=\left[\begin{array}{ccccc}
0 & 40 & 60 & 40 & 30 \\
40 & 0 & 40 & 60 & 30 \\
60 & 40 & 0 & 40 & 30 \\
40 & 60 & 40 & 0 & 30 \\
30 & 30 & 30 & 30 & 0
\end{array}\right]
$$

In this example, station $c_{5}=(20,20)$ is the resource station. Thus vector $r c$ becomes:

$$
r c=\left[\begin{array}{lllll}
0 & 0 & 0 & 0 & 1
\end{array}\right]
$$

We assume that the AGV has got an initial sequence of tasks it has to visit in a specified order.

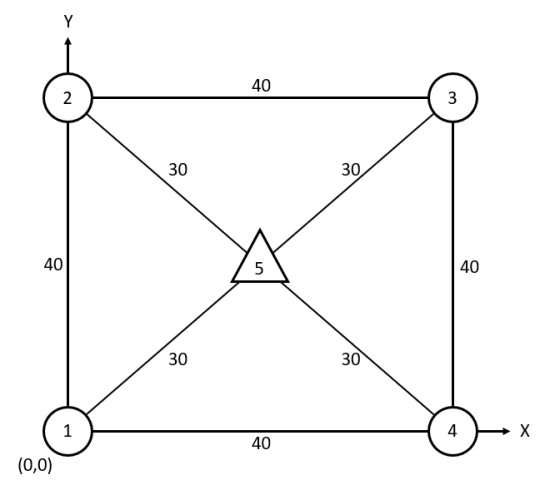

Figure 2: Situation

This tour is the input to our approach and is obtained by a TSP algorithm from a central or a decentral task allocation algorithm. This initial tour is represented as follows:

$$
\bar{x}=\left[\begin{array}{llll}
1 & 2 & 3 & 4
\end{array}\right]
$$

If the AGV starts its tour at the depot station, we assume that it has its maximum resource available $\left(R_{\text {init }}=\right.$ 
$\left.R_{\max }=100 \%\right)$. We also have to define the charging and consumption characteristics. We assume that the charging function is as follows:

$$
\operatorname{deltaR}_{c h}(T)=\left(\frac{R_{\max }}{T_{\max }}\right) \cdot T=\left(\frac{100}{100}\right) \cdot T=T
$$

This is an equation that represents the linear behaviour of the charging characteristic as mentioned in Section 4 with the use of realistic values for the example. After $T=T_{\max }=100 \mathrm{~s}$, the resources are full $\left(R=R_{\max }=\right.$ $100 \%)$. For the resource consumption, we assume that the characteristic is as follows:

$$
\operatorname{delta} R_{c}(t)=0.8 t
$$

This equation represents the dependency on time as mentioned in Section 4 also with the use of realistic values for the example. Finally, we simplify further by assuming that the AGV moves at a constant speed $v_{c}=1 \mathrm{~m} / \mathrm{s}$.

\subsection{Example problem evaluation}

We start with a first possible solution where the AGV chooses not to include any resources at all. Its tour thus remains unchanged. Matrix $r b c$ has all zero values.

$$
r b c=\left[\begin{array}{llll}
0 & 0 & 0 & 0
\end{array}\right]
$$

The solution tour becomes:

$$
\overline{\bar{x}}=\left[\begin{array}{llll}
1 & 2 & 3 & 4
\end{array}\right]
$$

In the solution, the AGV will also choose the time it will stay at the resources. But as it didn't chose any resource to visit, this does not matter. So we are not going to pay attention to the resource time vector $r t$.

When we evaluate the obtained solution, we can see that the AGV starts at station 1 with location $c_{1}=(0,0)$ with $R_{1}=R_{\text {init }}=100 \%$. The next stop on the tour is station 2 with location $c_{2}=(0,40)$. The AGV has to travel
$40 \mathrm{~m}$ with a speed of $1 \mathrm{~m} / \mathrm{s}$. The travel time between these stations is thus $40 \mathrm{~s}$. Using equation 15 we can compute the consumed resources delta $R_{c}=32 \%$. The remaining resource level at the second station on the solution tour is thus $R_{2}=100-32=68 \%$. We can calculate this for every station:

$$
\begin{gathered}
R_{3}=68-32=36 \% \\
R_{4}=36-32=4 \% \\
R_{5}=4-32=-28 \%
\end{gathered}
$$

Where $R_{i}$ is the $i^{\text {th }}$ station on the tour. We can see that the AGV cannot reach the last station from station 4 because its resources would be negative. This solution will thus be discarded due to our nonlinear resource constraint. To get to the end station, the AGV will definitely need to include a resource station.

If the AGV chooses to include a resource station after station 4 in its initial tour, then its matrix $r b c$ would be as follows:

$$
r b c=\left[\begin{array}{llll}
0 & 0 & 0 & 1
\end{array}\right]
$$

The total tour including charging station will then look like:

$$
\overline{\bar{x}}=\left[\begin{array}{lllll}
1 & 2 & 3 & 4 & 5
\end{array}\right]
$$

Besides the choice to insert a charging point at a particular position in the initial task sequence, the AGV can also choose how long it will charge to minimize total travel time. Let say that the AGV chooses to charge until its resource level reaches $80 \%$. This number may not be the optimal choice but is just chosen as a realistic value to obtain clear results in the example. Because $T_{R=80 \%}=$ $80 \mathrm{~s}$, the matrix $r t$ will become:

$$
r t=\left[\begin{array}{llll}
0 & 0 & 0 & 80
\end{array}\right]
$$

If we recapitulate, the resources at the first four stations in 
the tour would be the same. $R_{4}$ is the amount of available resources at the fourth station of the tour which is now station 4 .

$$
\begin{gathered}
R_{1}=100 \% \\
R_{2}=100-32=68 \% \\
R_{3}=68-32=36 \% \\
R_{4}=36-32=4 \%
\end{gathered}
$$

The process continues as follows:

- After station 4, the AGV includes resource station 5 to charge there.

- To go from station 4 to station 5 , this would take the $\mathrm{AGV}$ an amount of resources $R=0.8 \cdot 30=24 \%$ as the distance $d_{45}$ between station 4 and station 5 is $30 \mathrm{~m}$.

- We can see that the resources the AGV has left at station 4 is only $4 \%$. This is not enough to go to station 5. Because otherwise its resource level at station 5 would be negative $(4-24=-20 \%)$.

As a further modification of the solution, the AGV could instead choose to include the resource station after station 3 in its initial tour. The matrix $r b c$ then becomes:

$$
r b c=\left[\begin{array}{llll}
0 & 0 & 1 & 0
\end{array}\right]
$$

The total tour including charging station will then look like:

$$
\overline{\bar{x}}=\left[\begin{array}{lllll}
1 & 2 & 3 & 5 & 4
\end{array}\right]
$$

We will state again that the AGV will choose to stay at this resource station till its resources reached $80 \%$. Because $T_{R=80 \%}=80 \mathrm{~s}$, the matrix $r t$ will then become:

$$
r t=\left[\begin{array}{llll}
0 & 0 & 80 & 0
\end{array}\right]
$$

If we now make the calculations, we see that the AGV can reach the end station without any problems:

$$
\begin{gathered}
R_{1}=100 \% \\
R_{2}=100-32=68 \% \\
R_{3}=68-32=36 \% \\
R_{4}=36-24+80=92 \% \\
R_{5}=92-24=68 \% \\
R_{6}=68-32=36 \%
\end{gathered}
$$

We can see that the AGV arrived at the last station (starting station) with still enough resources left and thus stayed longer than necessary at the resource station. The total travel time is the sum of the total travel times between each station:

$$
(40+40+30+30+40)=180 s
$$

and the total time charged at resource stations is:

$$
\sum_{i=1}^{m}(r t \times r b c)=\sum_{i=1}^{1}\left(\left[\begin{array}{llll}
0 & 0 & 80 & 0
\end{array}\right] \times\left[\begin{array}{l}
0 \\
0 \\
1 \\
0
\end{array}\right]\right)=80 \mathrm{~s}
$$

This makes a total time of $260 \mathrm{~s}$. The total execution time of the whole tour could be reduced if the AGV would choose to stay not that long in the resource station. If for example its vector $r t$ would be:

$$
r t=\left[\begin{array}{llll}
0 & 0 & 50 & 0
\end{array}\right]
$$

Looking at the charging function, the total gained resource would be:

$$
\operatorname{delta}_{c h}(T)=\left(\frac{100}{100}\right) \cdot 50=50 \%
$$


Then the resources at each station would become:

$$
\begin{gathered}
R_{1}=100 \% \\
R_{2}=100-32=68 \% \\
R_{3}=68-32=36 \% \\
R_{4}=36-24+50=62 \% \\
R_{5}=62-24=38 \% \\
R_{6}=38-32=6 \%
\end{gathered}
$$

The AGV would still be able to reach its end station, but the total travel time is reduced to $230 \mathrm{~s}$. By reducing this charging time, the AGV executed its total tour in less time by avoiding extra unnecessary charging time. Another way to reduce charging time is to choose the charging station which is the closest to the initial tour. In this section we gave a simple example to clarify our approach. In general the AGV can include more resource stations into its tour and choose appropriate charging times at each resource visit. In the next section, a simulation of our approach will be made.

\section{Optimized example problem}

In this section, we will use our approach to obtain an optimal insertion of a charging point and an optimal charging time using the minimal total execution time of the tour as objective. Since the focus of this paper is to propose a feasible approach to the optimization of resource management in AGV systems, we will use the Particle Swarm Optimization as it is a robust and already used algorithm for similar problems [13, 14] and we leave the comparison of this algorithm with others to further research works.

\subsection{Example problem description}

The simulation assumes an operating area which consists of 9 normal stations $(n=9)$ and 3 charging stations $(m=3)$. The position of every station in the area is shown in Figure 3. Station 1 is the starting station and station

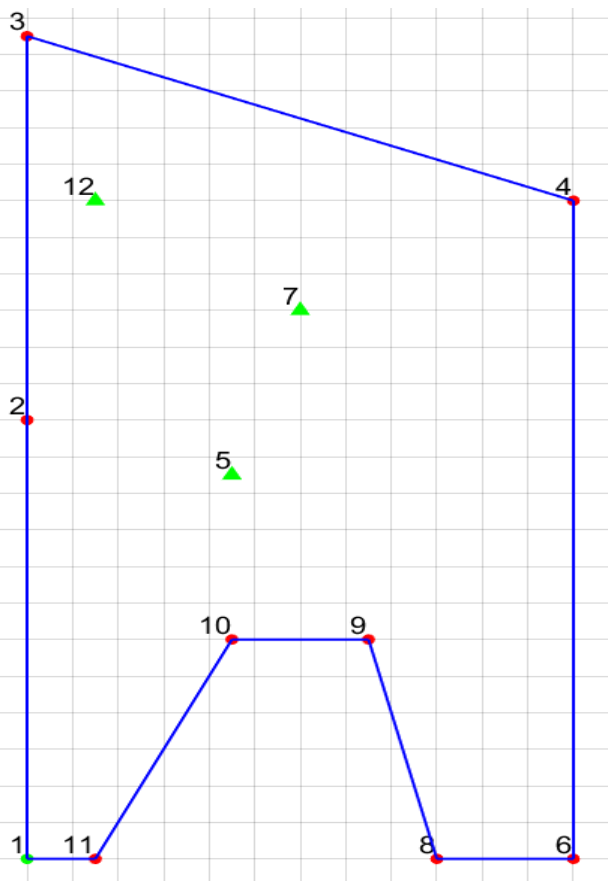

Figure 3: Initial tour

5, 7, and 12 are charging stations.

The locations of the stations are gathered in vector $c$ with length $(n+m)=12 . c_{1}=(0,0)$ is the start station.

$c=\left[\begin{array}{cccccccccrrr}0 & 0 & 0 & 24 & 9 & 24 & 12 & 18 & 15 & 9 & 3 & 3 \\ 0 & 24 & 45 & 36 & 21 & 0 & 30 & 0 & 12 & 12 & 0 & 36\end{array}\right]$

Vector $r c$ becomes:

$$
r c=\left[\begin{array}{llllllllllll}
0 & 0 & 0 & 0 & 1 & 0 & 1 & 0 & 0 & 0 & 0 & 1
\end{array}\right]
$$

We assume that the robot initially gets a sequence of locations it has to visit in a predefined order. In our simulation, tour $\bar{x}$ is presented as follows.

$$
\bar{x}=\left[\begin{array}{lllllllll}
1 & 2 & 3 & 4 & 6 & 8 & 9 & 10 & 11
\end{array}\right]
$$

The tour starts at the starting station and also ends there. This initial tour still does not contain any charging station. So given this tour, it can be possible that the robot is not able to finish the tour given an initial amount of resources.

We simulate a situation where the robot starts at the 
depot station and thus is fully charged $\left(R_{\text {init }}=100 \%\right)$. In Figure 4 the progress of the resource level during the tour without including charging stations is shown.

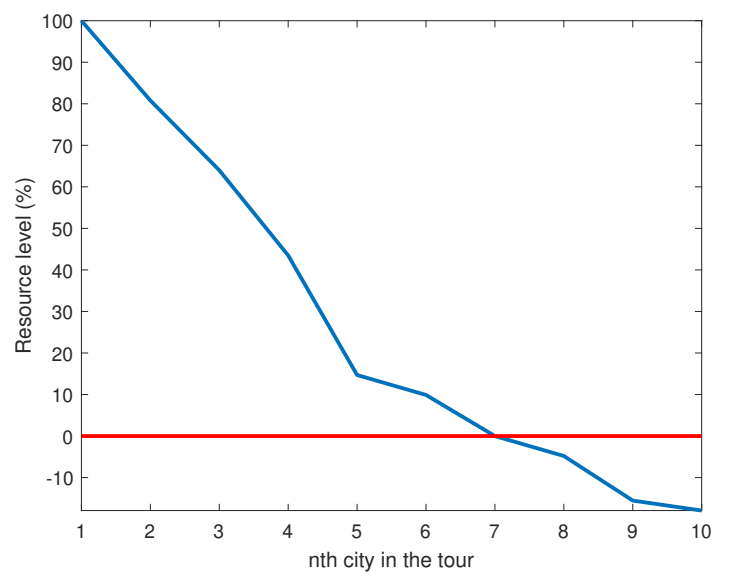

Figure 4: Resource level progress without charging stations

On the $x$-axis, the tour is presented where number 1 stands for the first station in the tour. There can be seen that the robot has not enough resources to complete the tour as the resource level drops below zero. So there is a need to include charging stations in the tour.

\subsection{Optimization setup}

Given this situation, we can use the Particle Swarm Optimization to find an optimal solution. The solution vector of the optimization contains the matrix $r b c$ of size $(n \times m)$ which denotes if a resource station is added between any two stations. $r b c_{i j}=1$ if charging station $\mathrm{j}$ is included after station i. The solution vector also contains a resource time matrix $r t$ of size $(1 \times n)$. This matrix denotes how long the robot will charge at the inserted charging location defined by matrix $r b c$. This makes a solution vector of length $(n \cdot m+n)$. The $r b c$ matrix can only contain binary values and the rt matrix can contain doubles from the minimum resource time $T_{\min }$ to the maximum resource time $T_{\max }$. For the simulation, we use the same charging and consumption characteristics as in Section 5 .

$$
\begin{gathered}
\operatorname{deltaR}_{c h}(T)=\left(\frac{100}{100}\right) \cdot T \\
\operatorname{deltaR}_{c}(t)=0.8 t
\end{gathered}
$$

It takes the robot 100 seconds to charge fully $\left(T_{\max }=\right.$ $100 \mathrm{~s})$. We assume a constant velocity of $1 \mathrm{~m} / \mathrm{s}\left(v_{c}=1\right)$.

The optimization uses a swarm size of 200 with a maximum of stall iterations of 1000 . These parameters are obtained after some trial-and-error and gave the best results. We make use of a hybrid optimization which executes an extra constrained nonlinear multi-variable optimization after the particle swarm optimization to improve the results. Also, an initial swarm matrix is defined where all variables equal zero, which means that no charging stations are included and that all resource times equal zero.

\subsection{Example problem evaluation}

If we run the Particle Swarm Optimization using previously defined setup parameters, we get the following solution values for matrix $r b c$ and matrix $r t$.

$$
r b c=\left[\begin{array}{ccc}
0 & 0 & 0 \\
0 & 0 & 1 \\
0 & 0 & 0 \\
0 & 0 & 0 \\
0 & 0 & 0 \\
0 & 0 & 0 \\
0 & 0 & 0 \\
0 & 0 & 0 \\
0 & 0 & 0
\end{array}\right] \quad r t=\left[\begin{array}{c}
29 \\
19 \\
3 \\
21 \\
13 \\
21 \\
94 \\
20 \\
33
\end{array}\right]
$$

From $r b c$, we can see that the third $(m=3)$ charging station (charging station 12) is included after the second $(n=2)$ station in the initial tour $\bar{x}$. If we look at the corresponding charging time in matrix $r t$, than we can see a charging time $r t_{2}=19 \mathrm{~s}$. If we put this in our charging function, we get an added resource:

$$
\operatorname{delta}_{c h}(T)=\left(\frac{100}{100}\right) \cdot 19=19 \%
$$

The output of the optimization is the optimized tour including the charging stations subjected to all our con- 
straints.

$$
\overline{\bar{x}}=\left[\begin{array}{lllllllllll}
1 & 2 & \mathbf{1 2} & 3 & 4 & 6 & 8 & 8 & 9 & 10 & 11
\end{array}\right]
$$

The optimized tour is visualized in Figure 5

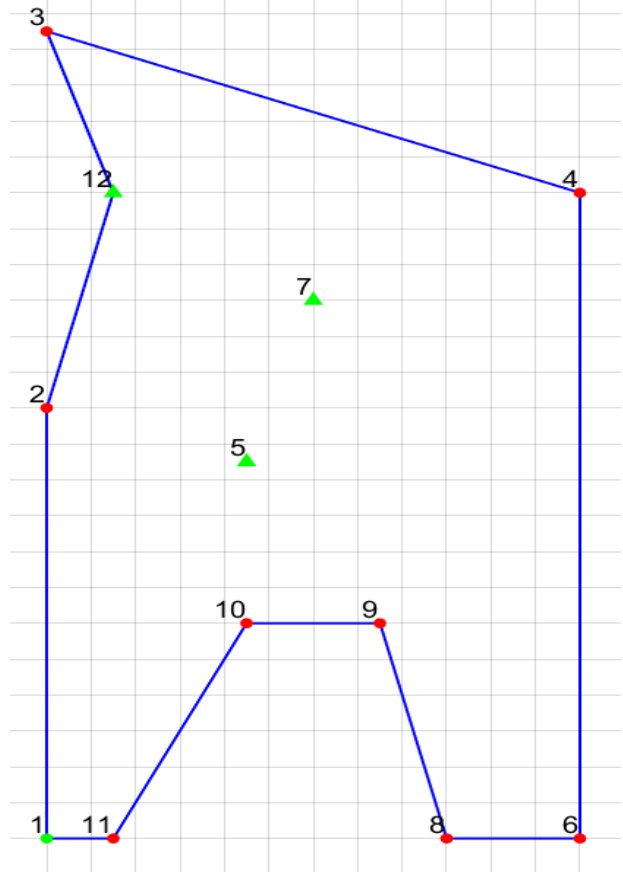

Figure 5: Optimized tour

Figure 6 shows the corresponding resource level progress during the tour. We can clearly see that the robot starts with an initial resource percentage of $100 \%$ at the depot station.

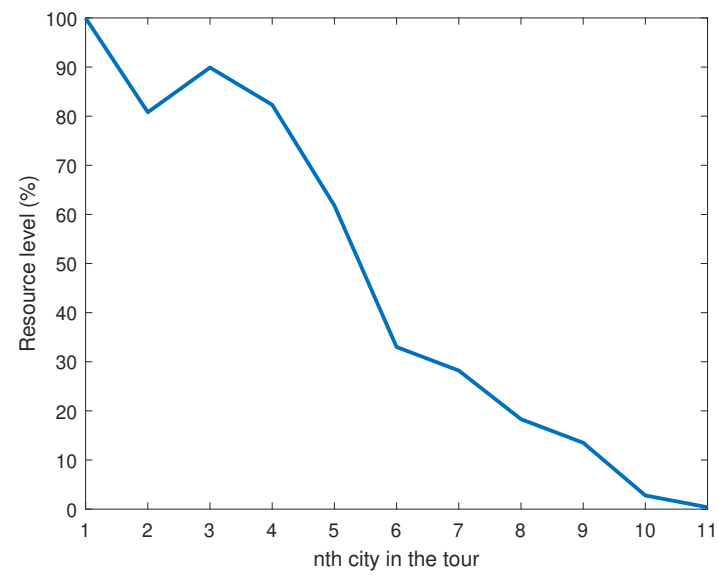

Figure 6: Resource level progress of the optimized tour

In charging station 12 (the third station in the tour), the resource level raises to $89.90 \%$. The resource level at the last station is zero. This means that the robot handles efficient resource management in which it can exactly complete its tour without having lost too much time charging. Once arrived at the depot station with this minimum amount of charging level, the AGV can again charge fully and start another tour from the depot station. The optimization outputs a total travel time, including charging at the inserted charging station of 167.27 seconds.

\subsection{Benchmark}

Now that we have an optimal solution following our approach, it would be interesting to compare this to a benchmark. As a benchmark, we take the current rulebased industrial practice where the robot keeps on driving until its resource level drops below a certain level. Then the robot heads to the closest charging station to charge fully. As threshold level, we take $20 \%$ as minimum State of Charge (SoC) according to H. Kiehne [11. We will use the exact same starting situation as in our previous simulation. After the simulation, we get the following tour:

$$
\overline{\bar{x}}=\left[\begin{array}{llllllllll}
1 & 2 & 3 & 4 & 7 & 6 & 8 & 9 & 10 & 11
\end{array}\right]
$$

The tour the robot would follow is shown in Figure 7 Figure 8 shows the corresponding resource level progress during the tour. We can see that the robot starts with an initial resource percentage of $100 \%$. In charging station 7 (fifth station on the tour), the resource level raises to the maximum of $100 \%$. The resource level at the last station is $41.52 \%$. This means that the robot still has a lot of resources left at the end of its tour. The robot thus spent too much time charging during the execution of its tour. The benchmark solution outputs a total travel time of 224.38 seconds. This is 57.11 seconds more than in our optimized approach. So we reduced the total traveling time including charging by $25 \%$ with respect to the benchmark. The main reasons for this reductions are (1) that the AGV will choose the most optimal charging point which is the point the closest to the tour, and (2) that the 
AGV will choose the most optimal charging time which is exactly the amount of time to end the tour with no resources left.

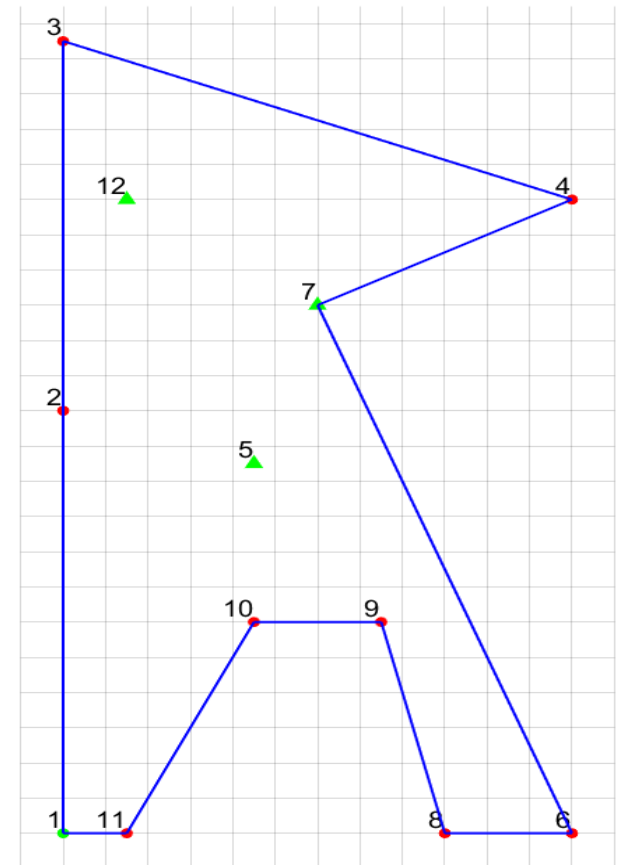

Figure 7: Benchmark tour

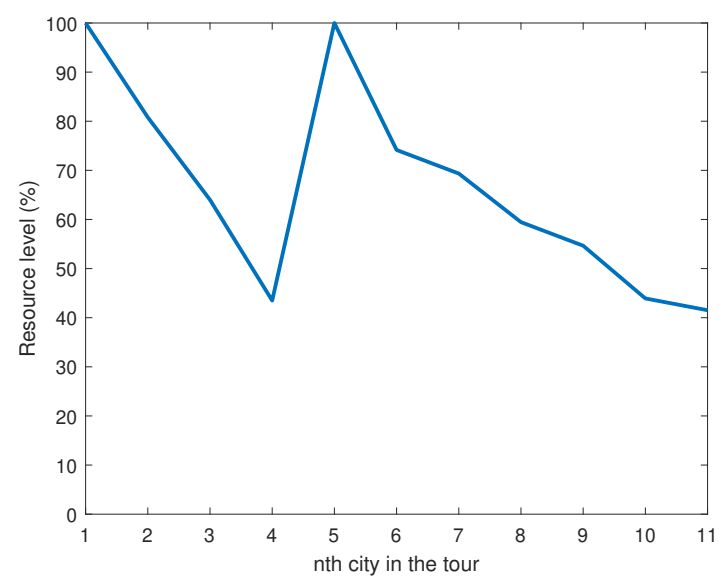

Figure 8: Resource level progress of the benchmark tour

\subsection{Results in function of the initial resource level}

The previous comparison between our approach and the benchmark was a comparison for 1 particular situation. But the behaviour of the resource management the robot handles is strongly dependent on the initial resource level the robot starts its tour with. In the beginning, we stated that the AGV always starts and ends at the depot station where it can charge fully. In this situation, the AGV always starts with a $100 \%$ battery level and can also charge to this level after finishing the tour. But in reality, it can be possible that the AGV starts at the depot station with less than a $100 \%$ battery level. The behaviour of both our approach and the benchmark will be different. So for this reason, in the following part, we will do the same comparison as in the previous section, but for initial resources in an interval from 0 to $100 \%$. The results are plotted in Figure 9

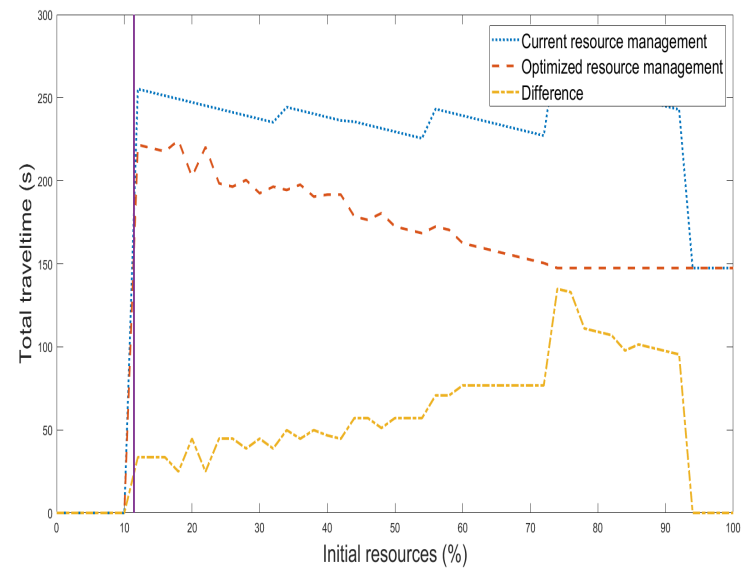

Figure 9: Total travel times in function of the initial resources for each approach

The vertical line on the left shows the minimum initial resource level needed to be able to reach the closest resource station from the starting station. If the initial resource level is lower than this limit, the robot is not able to start its tour and the total travel time is returned as zero. The dotted line shows the total travel time including charging times in function of the initial resource level for the benchmark approach. And the dashed line shows this for our approach. The dash-dotted line shows the difference between them. There can be clearly seen that our approach has a lower total travel time for each initial resource level. After a certain initial resource level of $94 \%$, both approaches reach the same total travel time which stays constant. At these levels, there are enough resources 
to complete the tour without visiting any charging station. The total travel time is thus the total time needed to complete the tour without visiting any other station. This is the reason why both approaches reach the same total travel time. Our approach already gets to this constant total time earlier than the benchmark (from $74 \%$ initial resource level). This is because, in the benchmark approach, the robot will still go to a charging station to charge fully after its resource level dropped below a certain level although it has enough resources to complete the tour. This is an extra feature where our approach seems to overtake the benchmark.

In Figure 10, the resource level progress during the tour for each approach at an initial resource level of 20, $40,60,80$, and $100 \%$ is plotted. There can be seen that in most cases, the benchmark is left with more resources after ending the tour then needed.
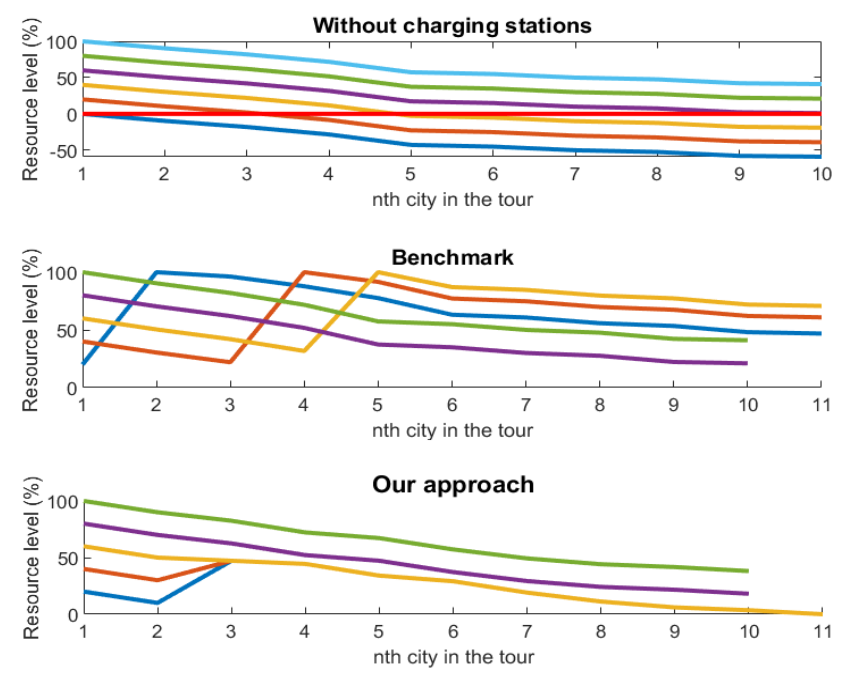

Figure 10: Resource progress during the tour for several initial resource levels

The figure also reveals that some tours end after visiting ten stations and some tours end after visiting eleven stations. In the tours visiting ten stations, no charging stations are added. In the tours visiting eleven stations, one charging station is added. Our approach will always end the tour with zero resources. Unless the initial re- sources are enough to complete the tour without charging, then both approaches are left with the same resource level. This shows that our approach handles very efficient resource management as it does not waste time on charging more than needed to complete the tour. The figure also shows that in our approach, the AGV will always choose to charge at charging station 12 (third station on the tour). This is the optimal choice as this charging station lays the closest to the initial tour.

\subsection{Remarks to the simulation}

The simulation assumes that the resource level of the AGV can vary between zero and $100 \%$. In our approach, the AGV even ends its tour with a resource level of zero, which is not practically interesting. As mentioned in Section 2, lots of research is done to an optimal charging scheme for the battery. The research of Q. S. Kabir et al. 3] showed that a higher frequency of charging will benefit manufacturing performance. According to Q. S. Kabir et al. 9], the most used batteries in AGVs are Valve Regulated Lead Acid (VRLA) batteries for which deep discharge is detrimental. It would thus be better to raise the batteries minimum charging level. The behaviour of not reaching the maximum resource level, is already present in our approach as the optimization algorithm will rather choose to charge less then $100 \%$ to minimize charging times. Figure 10 confirms that our approach never exceeds the limit of $80 \%$ unless the initial resource level is higher. On the other hand, our approach tries to end the tour with a resource level of zero. As this is detrimental for the life cycle of the battery, these limits can be adjusted in the simulation so that the level of the battery will never exceed these limits. If we adjust the minimum resource level to $20 \%$, our approach outputs a behaviour as visible in Figure 11. The horizontal lines show the lower limit of $20 \%$ and the upper limit of $80 \%$. It is clear that our approach now tries to reach this new value of $20 \%$ at the final station which shows very efficient resource management as there 


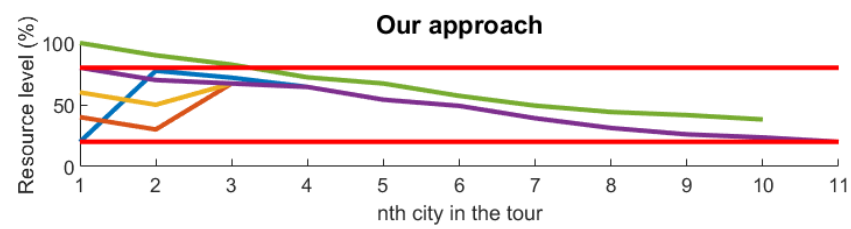

Figure 11: Resource progress during the tour for several initial resource levels with a lower resource limit of $20 \%$

is no more charging time spend than needed. By using this adaptation to our initial approach, the battery level always falls in between the boundaries to guarantee maximum battery lifetime. In general, every battery treatment that is required to maximize the 'health' of a battery can be included in the optimization. Maximizing the lifetime of a battery can, next to the minimization of total travel time, be a second optimization objective.

\section{Situation of our approach in a decentralized con- text}

The current industrial offered and deployed AGV systems almost all work centralized. This means that one single computer controls the whole fleet of AGVs. Inside this central entity, optimization is done considering global information. The central unit has access to the positions [15] and amount of resources of every vehicle in the area and has the position of every task which has to be executed. Using this information, the central unit can assign a set of tasks to each robot and compute the path for each robot to execute these tasks in the most optimal sequence. While doing this, it also considers and prevents deadlocks and collisions with other AGVs on the route. As this optimization is quite hard for a small number of vehicles, due to limited memory and limited computation time, practical problems occur in the current industrial systems. For this reason, we opt for a decentralized optimization. The goal is to let this optimization run on the AGV itself and not on the central computer. In this way, we shift some of the computation load from the limited central unit to the AGVs themselves. This decentralization of control is one of the key features to migrate towards a flexible manufacturing system [16]. We assume that the AGV receives an already optimized list of places in the factory it has to visit in a specified sequence without any charging stations. The AGV could have got this list from a centralized or a decentralized task allocation process. The task of the $\mathrm{AGV}$ is to insert charging stations into this tour in an optimal way. Hence, not the central unit but the AGV itself manages its resources. Independent on how many AGVs we implement this extra optimization, the central unit will not experience any difference.

Although this decentralized approach will cause benefits regarding the flexibility and scalability of the total system and will come to a local optimum, this will cause highly sub-optimal global solutions for the whole system. This is because, in our approach, an AGV inserts charging stations considering its own benefits and not the benefit of the whole system. Some problems which are a consequence of this selfish behaviour and which causes the sub-optimal solutions are listed below. We also propose future solutions to these problems.

- A first reason is a concurrence in the system because of multiple AGVs which each strive for their own goals. The solution of one AGV will be locally optimal, but will for the whole fleet, be highly sub-optimal. When using more robots which will strive for their local optimal solutions, there will occur some competition. Single charging stations will be occupied by more AGVs at the same time. This will affect the local optimum for each AGV. It will thus be necessary that the AGVs communicate with each other by reserving charging stations in an interval somewhere in the future and communicate this reservation to the other AGVs. Other AGVs can take this into account and find an optimal solution according to this extra constraint. This can be seen in Figure 12. $T_{i}$ is a certain task to be executed and 
$R_{i}$ is a charging station. The AGVs use the information of charging station reservations of the whole fleet and use it to optimally insert charging stations into their tour. They make use of sort of schedule board on which they can put their reservations. After the insertion, they provide information about their resource reservation to the other AGVs.

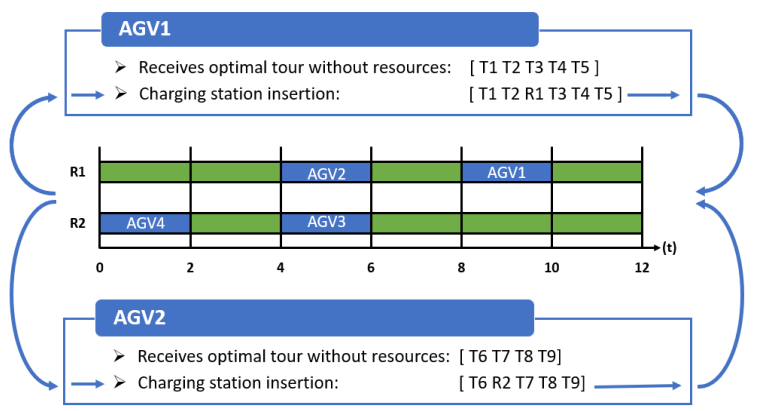

Figure 12: AGVs communicate their resource reservations

- A second reason is that it can be possible that the tour will not be optimal anymore after inserting the charging stations on the local level. It can be possible that it would be more optimal to switch the sequence of two locations in the tour knowing the extra information of the positions of the inserted charging stations. So after the insertion process of the charging stations, it would be beneficial if the AGV would further optimize this route locally. This concept can be seen in Figure 13. The optimization finds a better solution by swapping task 3 and task 4 .

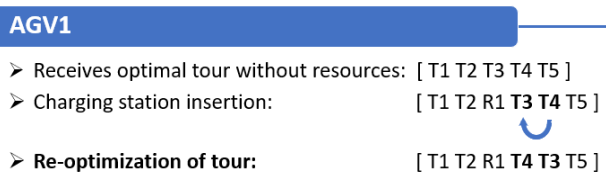

Figure 13: Extra local optimization after charging station insertion

- A last reason is when inserting the charging stations, a more sub-optimal route can be formed on the global level. It can be possible that if the central unit had known before which resources the AGV has inserted, that it would have assigned a whole other initial tour to the AGV. So it would be beneficial that after inserting the charging stations, and after doing a second optimization, that the AGV feeds the fitness of the new tour back to the global optimizer which maybe finds a better solution knowing this extra information. This concept can be seen in Figure 14. The global optimizer sends the optimized tour to the AGV, and the AGV sends the result of the optimized tour with charging stations back to the global optimizer. This will in its turn try to find a better solution given the information from the AGV.

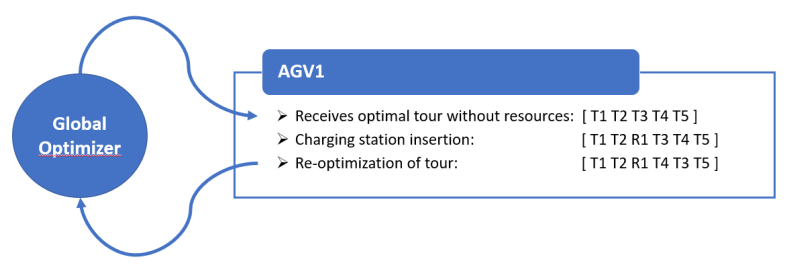

Figure 14: Feedback of resource insertion information to the central system

So to conclude these causes of sub-optimality, we can say that it would be too sub-optimal when doing this resource management fully decentralized without any communication with other AGVs and with the central unit. By feeding the decentral optimization result back to the central unit and to other AGVs, the global solution could be more optimal. We proposed an example of how this can be done by giving a solution concept for each cause of suboptimality. We will not state these concepts are the best for this application. In our following work, other ways of feedback, or other ways to communicate the whole system will be studied. But for now, we will consider the concepts described above.

\section{Conclusions}

This paper proposes an approach which will make resource management in industrial AGV systems more efficient. Literature reveals that battery management deci- 
sions do have an impact on the system performance, but is not conclusive on how to approach. Using our approach, AGVs will optimally include charging stations into their assigned tour and choose how long they will charge. Using a specific scenario and a Particle Swarm optimization, the total traveling time including charging was reduced significantly compared to the current resource management where AGVs charge fully when their resource level drops below a certain limit. Our approach strives for a minimal resource level at the end of the tour which indicates that the AGV managed its resources efficiently. Our approach assumes that the AGV knows how much it will consume between every station. Although more sophisticated charging and consumption characteristics can easily be implemented in the approach, we did not use complex consumption characteristics to keep our focus on the optimization of the insertion of charging stations. In reality, this battery consumption will depend on several factors like speed, weight, acceleration, friction, specific chemistry of the batteries, etc. In further research, we will also consider uncertainty involved in the problem. We can for example state that the amount of charging stations available is uncertain, or that the amount of gained resources in function of charging time is uncertain. We will demonstrate the results in our lab with a real application as our future works.

\section{Acknowledgements}

This work is supported by the M-group, part of the KU Leuven Campus in Bruges.

\section{References}

[1] J. Szpytko, P. Hyla, Automated Guided Vehicles Navigating Problem In Container Terminal, Logistics and Transport 2 (13) (2011) 107-116.

[2] B. Esmaeilian, S. Behdad, B. Wang, The evolution and future of manufacturing: A review, Journal of Manufacturing Systems 39 (2016) 79-100.
[3] Q. S. Kabir, Y. Suzuki, Increasing manufacturing flexibility through battery management of automated guided vehicles, Computers and Industrial Engineering 117 (January) (2018) $225-236$.

[4] M. De Ryck, M. Versteyhe, F. Debrouwere, Automated Guided Vehicle Systems, State-Of-The-Art Control Algorithms and Techniques (Submitted), Journal of Manufacturing Systems (2019).

[5] I. F.A. Vis, Survey of research in the design and control of automated guided vehicle systems, European Journal of Operational Research 170 (3) (2006) 677-709.

[6] T. Le-Anh, M. De Koster, A review of design and control of automated guided vehicle systems, European Journal of Operational Research 171 (1) (2006) 1-23.

[7] R. W. McHaney, Modelling battery constraints in discrete event automated guided vehicle simulations, International Journal of Production Research 33 (11) (1995) 3023-3040.

[8] M. Ebben, Logistic Control In Automated Transportation Networks, Ph.D. thesis (2001).

[9] Q. S. Kabir, Y. Suzuki, Comparative analysis of different routing heuristics for the battery management of automated guided vehicles, International Journal of Production Research 57 (2) (2018) 624-641.

[10] T. Kawakami, S. Takata, Battery Life Cycle Management for Automatic Guided Vehicle Systems, Design for Innovative Value Towards a Sustainable Society (2012) 403-408.

[11] H. Kiehne, Batteries for electrically powered industrial trucks, in: Battery Technology Handbook, 2nd Edition, Marcel Dekker Inc., New York, 2003, pp. 101-118.

[12] R. Matai, S. Singh, M. Mittal, Traveling Salesman Problem: an Overview of Applications, Formulations, and Solution Approaches, in: Traveling Salesman Problem, Theory and Applications, 2010.

[13] C. Guan, Z. Zhang, S. Liu, J. Gong, Multi-objective particle swarm optimization for multi-workshop facility layout problem, Journal of Manufacturing Systems 53 (2019) 32-48.

[14] S. D. Gulcu, H. K. Ornek, Solution of Multiple Travelling Salesman Problem using Particle Swarm Optimization based Algorithms, International Journal of Intelligent Systems and Applications in Engineering 7 (2) (2019) 72-82.

[15] S. Lu, C. Xu, R. Y. Zhong, L. Wang, A RFID-enabled positioning system in automated guided vehicle for smart factories, Journal of Manufacturing Systems 44 (2017) 179-190.

[16] I. Baffo, G. Confessore, G. Stecca, A decentralized model for flow shop production with flexible transportation system, Journal of Manufacturing Systems 32 (2013) 68-77. 